TITLE:

\title{
Cauer ladder network representation of a nonlinear eddy- current field using a first-order approximation
}

\author{
$\operatorname{AUTHOR}(\mathrm{S})$ :
}

Eskandari, Hamed; Matsuo, Tetsuji

\section{CITATION:}

Eskandari, Hamed ...[et al]. Cauer ladder network representation of a nonlinear eddycurrent field using a first-order approximation. IEEE Transactions on Magnetics 2020, 56(2): 7505904.

\section{ISSUE DATE:}

2020-02

URL:

http://hdl.handle.net/2433/245886

\section{RIGHT:}

(c) 2020 IEEE. Personal use of this material is permitted. Permission from IEEE must be obtained for all other uses, in any current or future media, including reprinting/republishing this material for advertising or promotional purposes, creating new collective works, for resale or redistribution to servers or lists, or reuse of any copyrighted component of this work in other works.; この論文は出版社版でありません。引用の際には出版社版をご確認ご利用ください。; This is not the published version. Please cite only the published version. 


\title{
Cauer Ladder Network Representation of a Nonlinear Eddy-current Field using a First-order Approximation
}

\author{
Hamed Eskandari ${ }^{1}$, Tetsuji Matsuo ${ }^{1}$ \\ ${ }^{1}$ Graduate School of Engineering, Kyoto University, Kyoto 615-8510, Japan
}

\begin{abstract}
An approximation approach is proposed for the analysis of nonlinear quasi-static eddy currents adopting a Cauer ladder network (CLN). When there are nonlinear magnetic materials in the analysis domain, the electric and magnetic modes and their corresponding values for resistors and inductors in the CLN may vary according to the level of saturation in the core. Considering the effects of the modes and their magnitudes on the saturation imposes a heavy computational burden, which brings the efficiency of the CLN method into question. This paper studies a first-order approximation of the nonlinear CLN method to keep the nonlinearization procedure computationally effective, simple, and accurate. Numerical tests are carried out for a two-dimensional nonlinear inductor excited with rectangular and sinusoidal excitations to show the accuracy of the proposed nonlinear resolution method.
\end{abstract}

Index Terms - Nonlinear Cauer Circuit, eddy current, finite element method, model order reduction.

\section{INTRODUCTION}

$\mathbf{E}$ LECTROMAGNETIC drive systems require accurate and computationally inexpensive mathematical models for their electromagnetic devices. These models are expected to cover all the moving parts, magnetic nonlinearities, eddy currents, and hysteresis losses over a wide range of frequencies. Although the finite element method (FEM) covers all the requirements mentioned above, its high computational cost makes it ineffective and less favorable in control applications.

The demand for efficient mathematical models on the one hand and the inadequate performance of the FEM on the other hand has encouraged researchers to apply model order reduction (MOR) techniques to magnetodynamic fields. MOR helps reduce the complexity of mathematical models without considerably sacrificing accuracy.

Among different MOR techniques, proper orthogonal decomposition (POD) is most commonly used in the field of computational electromagnetics [1]-[3]. POD-based MOR has been shown to be efficient for linear problems; however, it encounters difficulties in terms of a loss of accuracy in saturated regions, instabilities in numerical solutions, and increased computational cost. Innovative approaches have been proposed to tackle these issues [4]-[6]; however, the present paper solves the problem using a totally different approach based on the Cauer ladder network (CLN).

The CLN was originally proposed as an equivalent circuit representation of laminated magnetic sheets [7]. Owing to its strong physical interpretation and high accuracy, the CLN has been generalized beyond one-dimensional problems [8],[9]. The CLN can be constructed in a few sets of sequential finiteelement magnetostatic-field analyses. The use of the CLN method removes the need for taking snapshots and finding the eigenvalues of large matrices.

The present paper expands the applicability of the CLN to the nonlinear eddy-current field. The proposed method

Manuscript received December 1, 2012; revised August 26, 2015. Corresponding author: H. Eskandari (email: hamed.eskandari.28x@st.kyotou.ac.jp).

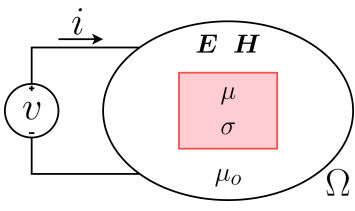

Fig. 1. Eddy-current field configuration

considers magnetic saturation by approximating the saturation effects in the first magnetic mode. The remainder of the paper is organized as follows. The second chapter briefly explains the CLN method. The third chapter discusses the effects of nonlinear magnetic material on CLN parameters. The fourth chapter proposes our nonlinearization technique and gives numerical examples.

\section{Matrix-Based Formulation of the Linear CLN}

In finite element space, the vector potential $\boldsymbol{A}$, electric field $\boldsymbol{E}$, and magnetic flux $\boldsymbol{B}$ can be defined with edge elements $\boldsymbol{w}_{i}^{1}$ and facial elements $\boldsymbol{w}_{j}^{2}$ as

$$
\boldsymbol{A}=\sum_{i} a_{i} \boldsymbol{w}_{i}^{1}, \quad \boldsymbol{E}=\sum_{i} e_{i} \boldsymbol{w}_{i}^{1}, \quad \boldsymbol{B}=\sum_{j} b_{j} \boldsymbol{w}_{j}^{2}
$$

in which $a_{i}$ and $e_{i}$, respectively, represent the line integration of $\boldsymbol{A}$ and $\boldsymbol{E}$ along the $i^{t h}$ edge while $b_{j}$ is the surface integration of $\boldsymbol{B}$ over the $j^{\text {th }}$ face in analysis domain $\Omega$ [10]. The set of line and face integrals are used to construct variable vectors as

$$
\boldsymbol{a}=\left[a_{1}, a_{2}, \ldots\right]^{T}, \boldsymbol{e}=\left[e_{1}, e_{2}, \ldots\right]^{T}, \boldsymbol{b}=\left[b_{1}, b_{2}, \ldots\right]^{T} .
$$

In the matrix formulation of the FEM, the curl operator is defined as an edge-face incident matrix, $C$, such that $\boldsymbol{B}=$ $\nabla \times \boldsymbol{A}$ can be rewritten as

$$
b=C a .
$$




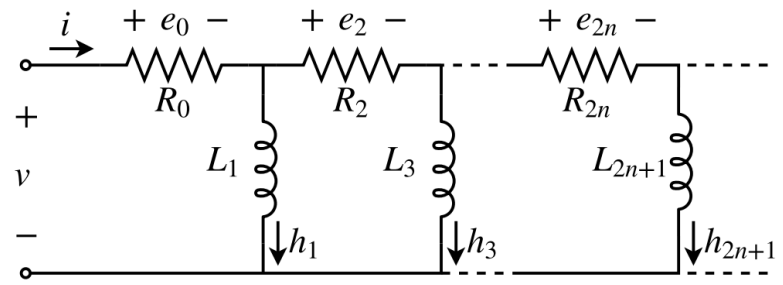

Fig. 2. Cauer circuit

The governing equation of the eddy-current field in quasimagnetostatic mode excited by the power supply as shown in Fig. 1 is

$$
\begin{aligned}
\boldsymbol{C}^{T} \boldsymbol{\nu} \boldsymbol{b} & =\boldsymbol{C}^{T} \boldsymbol{\nu} \boldsymbol{C a}=\boldsymbol{\sigma} \boldsymbol{e}+\boldsymbol{j}_{0} \\
\boldsymbol{C} \boldsymbol{e} & =-\partial_{t} \boldsymbol{b}=-\partial_{t} \boldsymbol{C a} .
\end{aligned}
$$

Here, $\boldsymbol{j}_{0}$ is the external current and $\boldsymbol{\nu}$ and $\boldsymbol{\sigma}$ are, respectively, the reluctivity and conductivity matrices defined as

$$
\begin{array}{ll}
\boldsymbol{\nu}=\nu_{i j}, & \nu_{i j}=\int_{\Omega} \frac{1}{\mu} \boldsymbol{w}_{i}^{2} \cdot \boldsymbol{w}_{j}^{2} d \Omega, \\
\boldsymbol{\sigma}=\sigma_{i j}, & \sigma_{i j}=\int_{\Omega} \sigma \boldsymbol{w}_{i}^{1} \cdot \boldsymbol{w}_{j}^{1} d \Omega,
\end{array}
$$

where $\mu$ and $\sigma$ are, respectively, the permeability and conductivity. For the sake of brevity, the coefficient matrix in (4) is denoted $\boldsymbol{K}$ :

$$
\boldsymbol{K}=\boldsymbol{C}^{T} \boldsymbol{\nu} \boldsymbol{C}
$$

The eddy-current field in Fig. 1 is described by its impedance in the frequency domain. The impedance can be represented in the form of continued fractions as

$$
\begin{aligned}
Z(s) & =\frac{V(s)}{I(s)}=\frac{b_{0}+b_{1} s+b_{2} s^{2}+\ldots}{a_{0}+a_{1} s+a_{2} s^{2}+\ldots} \\
& =R_{0}+\frac{1}{\frac{1}{L_{1} s}+\frac{1}{R_{2}+\frac{1}{\frac{1}{L_{3} s}+\ldots}}} .
\end{aligned}
$$

The equivalent circuit for (8) is depicted in Fig. 2. In the CLN method, the electric field $e$ and magnetic vector potential $\boldsymbol{a}$ are decomposed into a set of time-constant electric $\boldsymbol{e}_{2 n}$ and magnetic $\boldsymbol{a}_{2 n+1}$ modes, respectively weighted by $e_{2 n}$ and $h_{2 n+1}[8]$ :

$$
\begin{aligned}
& \boldsymbol{e}=\sum_{n=0}^{\infty} e_{2 n}(t) \boldsymbol{e}_{2 n}, \\
& \boldsymbol{a}=\sum_{n=0}^{\infty} h_{2 n+1}(t) \boldsymbol{a}_{2 n+1} .
\end{aligned}
$$

Referring to Fig. 2, $e_{2 n}$ and $h_{2 n+1}$ are, respectively, the voltage drop over $R_{2 n}$ and the current in $L_{2 n+1} . R_{2 n}$ and $L_{2 n+1}$ are, respectively, normalizing constants that ensure the orthogonality of the electric and magnetic modes:

$$
\begin{aligned}
\boldsymbol{e}_{2 n}^{T} \boldsymbol{\sigma} \boldsymbol{e}_{2 m} & =\frac{\delta_{n m}}{R_{2 n}}, \\
\boldsymbol{a}_{2 n+1}^{T} \boldsymbol{K} \boldsymbol{a}_{2 m+1} & =\delta_{n m} L_{2 n+1},
\end{aligned}
$$

where $\delta_{n m}$ is the Kronecker delta.
The CLN procedure starts from a unit power source and $\boldsymbol{a}_{-1}=0$ with the recurrence formulas

$$
\begin{aligned}
\boldsymbol{K}\left(\boldsymbol{a}_{2 n+1}-\boldsymbol{a}_{2 n-1}\right) & =R_{2 n} \boldsymbol{\sigma} \boldsymbol{e}_{2 n}, \\
\boldsymbol{e}_{2 n+2}-\boldsymbol{e}_{2 n} & =-\frac{1}{L_{2 n}} \boldsymbol{a}_{2 n+1} .
\end{aligned}
$$

\section{CLN with a Nonlinear Magnetic Core}

The linearization of the nonlinear magnetic core was also studied in [8]. The magnetic field in the core oscillates closely around a specific working point, and permeabilities are thus stored and frozen at that particular working point. It has been shown that better results are obtained with the differential frozen permeabilities $(d B / d H)$ than with the frozen permeabilities $(B / H)$. We therefore rely on differential permeabilities and reluctivities.

When there is a nonlinear magnetic core in $\Omega$, the reluctivity matrix should be considered an $\boldsymbol{a}$-dependent function:

$$
\boldsymbol{\nu}=\boldsymbol{\nu}^{\text {diff }}(\boldsymbol{B})=\boldsymbol{\nu}^{\text {diff }}(\boldsymbol{C} \boldsymbol{a}),
$$

where $\nu^{\text {diff }}$ is a differential reluctivity matrix that is the same as that in (6) except that $1 / \mu$ is the differential reluctivity as $d H / d B$. According to (10) and (15), the coefficient matrix in (7) can be rewritten as

$$
\begin{aligned}
\boldsymbol{K} & =\boldsymbol{C}^{T} \boldsymbol{\nu}^{\mathrm{diff}}(\boldsymbol{C} \boldsymbol{a}) \boldsymbol{C} \\
& =\boldsymbol{C}^{T} \boldsymbol{\nu}^{\mathrm{diff}}\left(\sum_{n=0}^{\infty} h_{2 n+1}(t) \boldsymbol{C} \boldsymbol{a}_{2 n+1}\right) \boldsymbol{C} .
\end{aligned}
$$

The proposed approach of approximating (4) is to consider the first mode as the only source of saturation in the magnetic core:

$$
\boldsymbol{K} \approx \boldsymbol{K}_{\boldsymbol{a}_{1}}^{\prime}=\boldsymbol{C}^{T} \boldsymbol{\nu}^{\text {diff }}\left(h_{1}(t) \boldsymbol{C} \boldsymbol{a}_{1}\right) \boldsymbol{C} .
$$

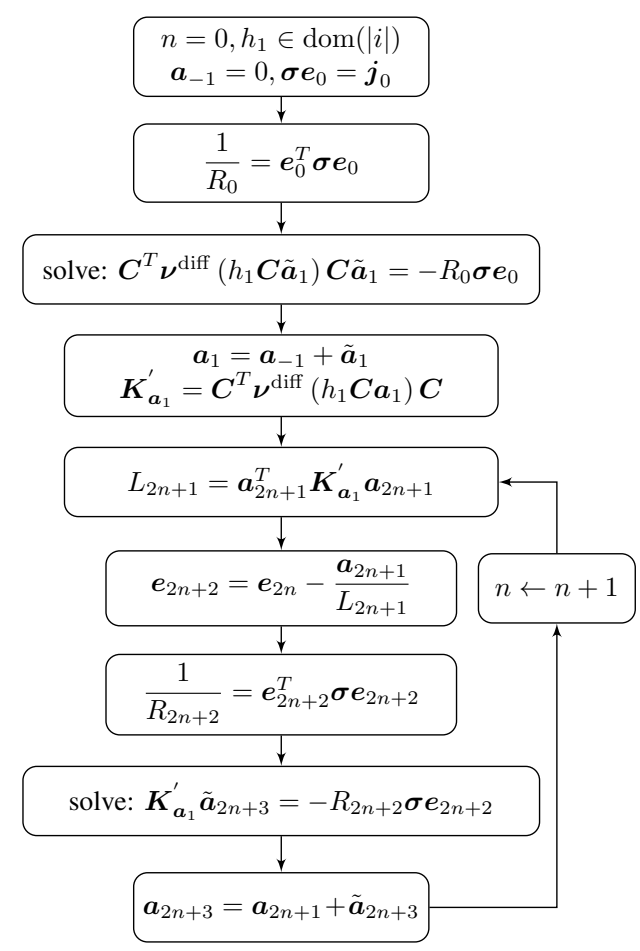

Fig. 3. Flowchart of the first-order nonlinear CLN procedure 


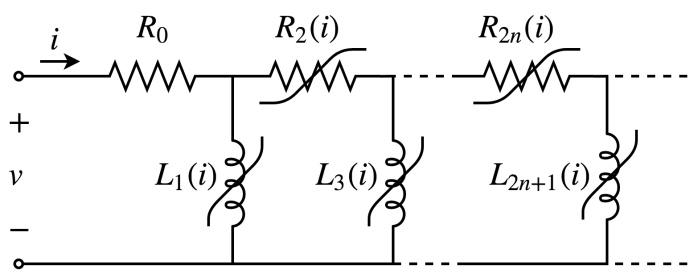

Fig. 4. First-order nonlinear CLN circuit

The first magnetic mode is the most dominant mode in shaping the saturation, and the above approximation will therefore lead to reasonable results. Similar to the nonlinearization approach used in [8], the reluctivities corresponding to $h_{1} \boldsymbol{a}_{1}$ are stored for higher-order modes. Thereafter, the shape and intensity of the electric and magnetic modes and their corresponding resistance and inductance values in the CLN will depend on $h_{1}$. The proposed procedure of the nonlinear CLN is depicted in Fig. 3. The procedure should be repeated for an adequate number of points in the desired range of $h_{1}$, to obtain look-up tables for $R_{2 n}\left(h_{1}\right)$ and $L_{2 n+1}\left(h_{1}\right)$.

The approximation applied in (17) implies that $h_{1}$ is the only factor affecting saturation. However, in practice, saturation in the core is affected by all $h_{2 n+1}$ components, equivalent to the total current, $i$. We therefore argue that

$$
\begin{aligned}
R_{2 n}\left(h_{1}\right) & \approx R_{2 n}(i), \\
L_{2 n+1}\left(h_{1}\right) & \approx L_{2 n+1}(i) .
\end{aligned}
$$

Finally, the nonlinear CLN model comprises $i$ dependent look-up tables for CLN parameters as depicted in Fig. 4.

\section{Computational Results}

The proposed model is applied to the two-dimensional ironcore inductor depicted in Fig. 5 [9] to evaluate its efficacy. Owing to geometrical symmetry, only one quarter of the domain is analyzed. The bulk-type iron core has a nonlinear magnetization characteristic defined by

$$
B=B_{s}\left(\operatorname{Coth}\left(\frac{H}{H_{s}}\right)-\frac{H_{s}}{H}\right),
$$

where $B_{s}=1.25 \mathrm{~T}$ and $H_{s}=544 \mathrm{~A} / \mathrm{m}$ are, respectively, the magnetic flux density and field intensity at a weak-saturation point. The conductivity of the conductor bar and the core are given by $4 \times 10^{7} \mathrm{~S} / \mathrm{m}$ and $1 \times 10^{6} \mathrm{~S} / \mathrm{m}$, respectively.

The core has single-turn coils excited with rectangular and sinusoidal signals having a frequency of $500 \mathrm{~Hz}$. The amplitudes of both excitations are set to 3 volts to guarantee adequate saturation in the core.

Saturation changes the shape and magnitudes of modes as discussed in section III. Figure 6 depicts the effect of saturation in the first magnetic mode on the higher-order modes. For the sake of clarity, the magnitudes of the modes are normalized according to maximum values. To represent the saturation of the magnitudes of the electric and magnetic modes, corresponding values of $R_{2 n}$ and $L_{2 n+1}$ are drawn with respect to $h_{1}$ in Fig. 7 .

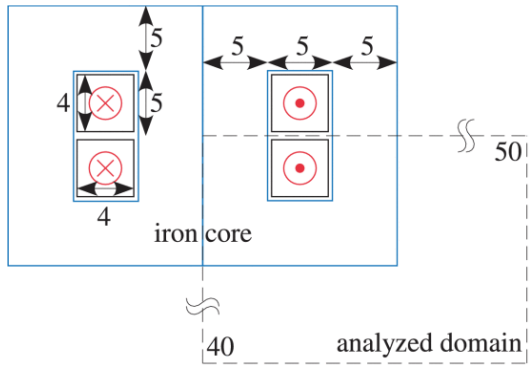

Fig. 5. Iron-core dimensions in millimeters

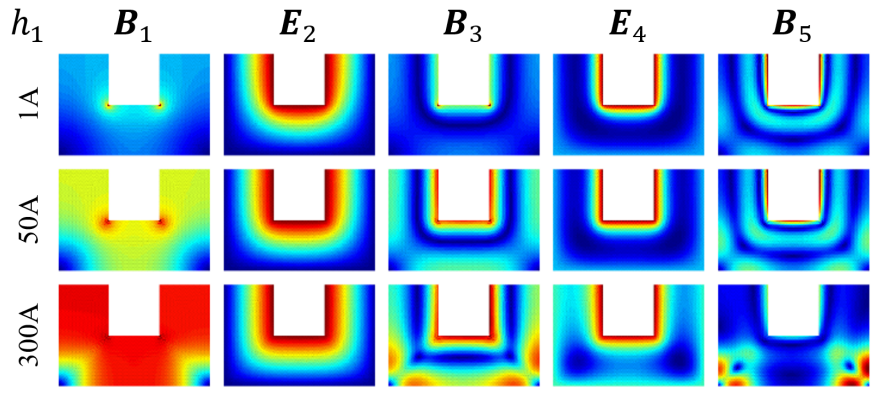

Fig. 6. Normalized electric and magnetic modes at different saturation points ( $h_{1}=1 \mathrm{~A}$ for the linear case, $h_{1}=50 \mathrm{~A}$ as for the knee-point and fully saturated regimes, $h_{1}=300 A$ )
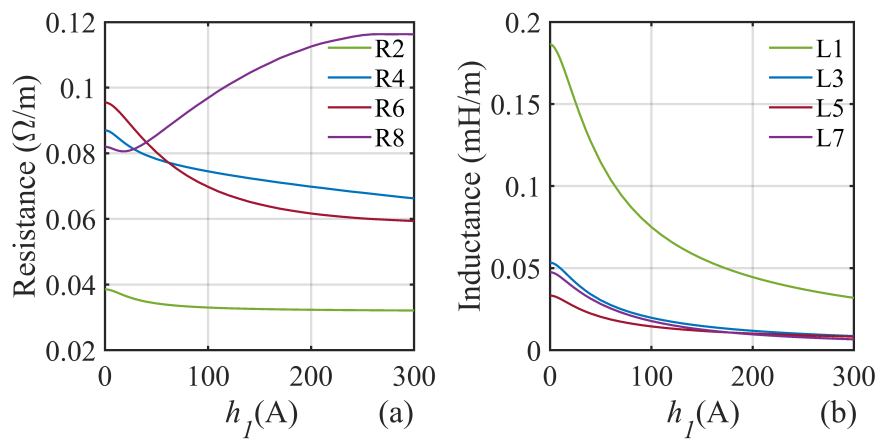

Fig. 7. (a) Resistances and (b) inductances of the nonlinear CLN circuit

\section{A. Transient Analysis}

To verify the proposed linearization method, the transient response of the eddy-current problem is studied over five cycles to cover both the transient and steady-state responses. The finite element mesh space has 137,042 elements with 69,022 nodes, and a three-stage resistive termination circuit is chosen for the CLN network model. In Fig. 8 and Fig. 9, the FEM and the nonlinear CLN driven transient responses are illustrated together with the linear CLN results. A comparison of the linear and nonlinear CLN responses shows that the input voltage is high for the core to saturate. Additionally, the nonlinear CLN network has acceptable error according to the results obtained using the FEM.

\section{B. Number of Stages}

In the CLN representation of the eddy-current field, a higher number of stages improves the accuracy of the transient response in higher-order harmonics. The same rule applies for the nonlinear CLN. 


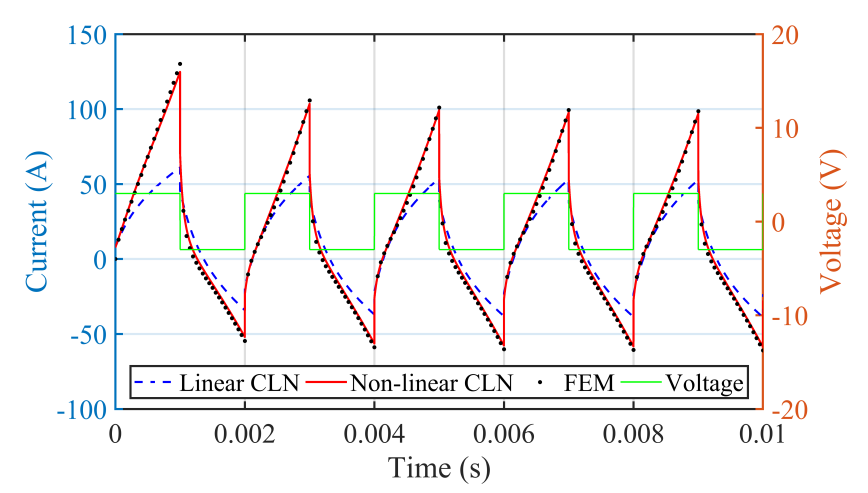

Fig. 8. Transient response with rectangular excitation

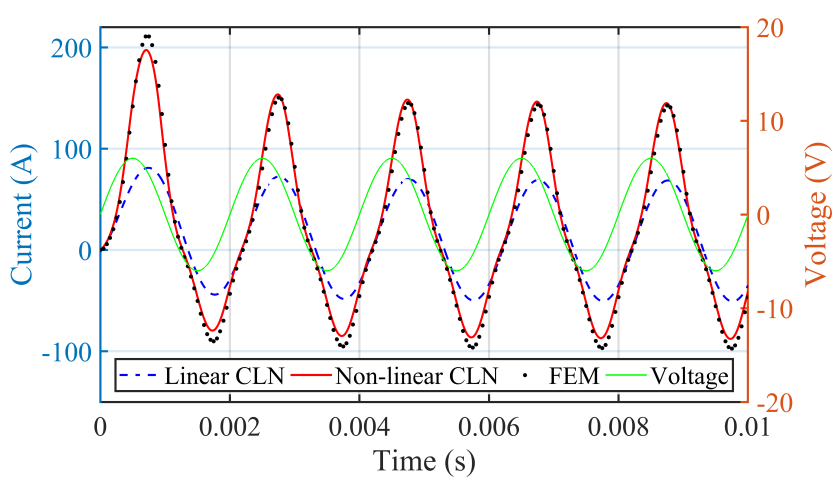

Fig. 9. Transient response with sinusoidal excitation

However, because the saturation is mainly determined by the modes with larger magnitudes (i.e., the first and third modes), the accuracy is nonlinearity becomes less dependent on the number of stages; see Fig. 10.

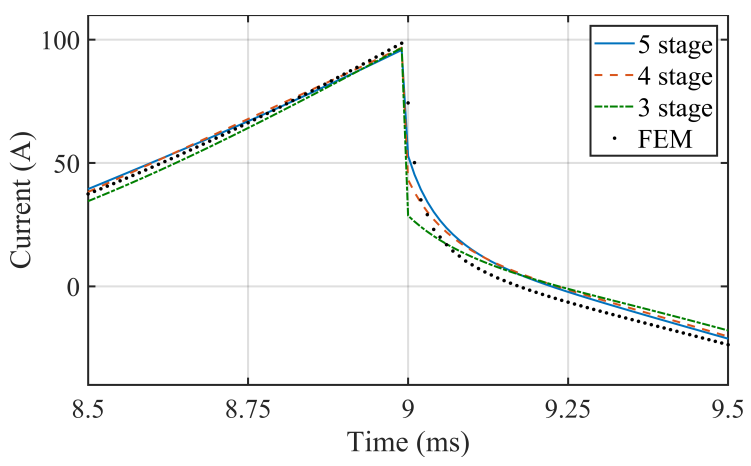

Fig. 10. Transient response with sinusoidal excitation

\section{Speed-up ratio}

Considering that the computational cost of transient analysis depends on the time span and time step, it is less meaningful to consider the speedup ratio of the proposed method and transient analysis. Similar to other MOR methods, the nonlinear CLN representation of the eddy-current field requires offline calculations to be made to obtain the CLN parameters. The offline computation of the proposed method comprises linear and nonlinear magnetostatic field calculations. The cost of the latter calculations is determined by the number of desired discrete values for $h_{1} \in \operatorname{dom}(|i|)$, where $\operatorname{dom}(|i|)$ is the range of all possible values for $|i|$.
In the case of the results shown in Fig. 8 and Fig. 9, the conventional time-stepping finite element analysis comprises 10 cycles with 100 time steps in each cycle. There are thus 1000 nonlinear field simulations. On the CLN side, a four-stage first ladder network is chosen with 10 points in $\operatorname{dom}(|i|)=[0,200]$ A. Ten nonlinear simulations are thus required for the first stage and $3 \times 10$ linear simulations for the remaining stages. Assuming that each nonlinear system of equations requires 4 iterations on average, the speed-up ratio is roughly approximated as

$$
\underset{\text { Speed-up }}{\text { Ratio }} \approx \frac{1000_{\text {nonlin }}}{10_{\text {nonlin }}+30_{\text {lin }}} \approx \frac{1000 \times 4}{10 \times 4+30}=57 .
$$

\section{CONCLUSion}

A first-order approximation method for the analysis of nonlinear eddy-current problems using the CLN concept was proposed. This method considers the saturation effect on the CLN parameters, considering only the first magnetic mode as the source of nonlinearization. The first magnetic mode is the most dominant, and the proposed approximation approach thus provides acceptable outcomes. Additionally, implementation of the proposed approach was simple and computationally inexpensive. Finally, the method was applied to a simple twodimensional nonlinear iron core to verify its accuracy. The model can be extended to consider both the first and second magnetic modes as the source of saturation in future work.

\section{ACKNOWLEDGMENT}

H. Eskandari gratefully acknowledges financial and invaluable moral support from the Yoshida Scholarship Foundation. The authors express their gratitude to Dr. H. Ebrahimi for his insightful and meticulous comments and Glenn Pennycook, MSc, from Edanz Group (www.edanzediting.com/ac) for editing a draft of this manuscript.

\section{REFERENCES}

[1] T. Henneron \& S. Clenet, "Proper Generalized Decomposition Method Applied to Solve 3-D Magnetoquasi-Static Field Problems Coupling With External Electric Circuits," IEEE Trans. on Magnetics, vol. 51, no. 6, Art. No. 7208910, Jun. 2015.

[2] T. Shimotani, Y. Sato, \& H. Igarashi, "Equivalent-Circuit Generation From Finite-Element Solution Using Proper Orthogonal Decomposition," IEEE Trans. on Magnetics, vol. 52, no. 3, Art. No. 15786633, 2016.

[3] D. Schmidthausler \& M. Clemens: "Low-order electroquasistatic field simulations based on proper orthogonal decomposition," IEEE Trans. Mag. vol. 48, no. 2, pp. 567-570, Feb. 2012.

[4] Y. Sato, T. Shimotani \& H. Igarashi, "Synthesis of Cauer-Equivalent Circuit Based on Model Order Reduction Considering Nonlinear Magnetic Property," IEEE Trans. on Magnetics, vol. 53, no. 6, Jun. 2017.

[5] R. Hasan, L. Montier, T.Henneron \& R. Sabariego, "Stabilized Reduced Order Model of a Non-Linear Eddy Current Problem by a Gappy-POD Approach,” IEEE Trans. on Magnetics, vol. 54, no. 12, pp. 1-8 Dec. 2018.

[6] T. Henneron \& S. Clenet, "Model Order Reduction of Non-Linear Magnetostatic Problems Based on POD and DEI Methods," IEEE Trans. on Magnetics, vol. 50, no. 2, pp. 33-36, 2014.

[7] Y. Shindo, T. Miyazaki \& T. Matsuo, "Cauer circuit representation of the homogenized eddy-current field based on the Legendre expansion for a magnetic sheet," IEEE Trans. Magnetics, vol. 52, no. 3, Art. no. 6300504, Mar. 2016.

[8] A. Kameari, H. Ebrahimi, K. Sugahara \& T. Matsuo, "Cauer Ladder Network Representation of Eddy-Current Fields for Model Order Reduction Using Finite-Element Method," IEEE Trans. on Magnetics, vol. 54, no. 3, Art. No. 7201804, Mar. 2018.

[9] T. Matsuo, A. Kameari, K. Sugahara \& Y. Shindo, "Matrix Formulation of the Cauer Ladder Network Method for Efficient Eddy-Current Analysis," IEEE Trans. on Magnetics, vol. 54, no. 11, Art. No. 7205805, Nov. 2018.

[10] A. Bossavit, Computational Electromagnetism, San Diego, CA, USA:Academic Press, 1998. 